\title{
An Ultra-Wideband (UWB) Ad Hoc Sensor Network for Real-time Indoor Localization of Emergency Responders
}

\author{
Anthony Lo ${ }^{1}$, Alexander Yarovoy ${ }^{1}$, Timothy Bauge 2 , Mark Russell ${ }^{2}$, \\ Dave Harmer2 and Birgit Kull ${ }^{3}$ \\ ${ }^{1}$ Delft University of Technology, \\ ${ }^{2}$ Thales Research \& Technology Limited, \\ IMST GmbH, \\ ${ }^{1}$ The Netherlands \\ 2UK \\ ${ }^{3}$ Germany
}

\section{Introduction}

A localization system is a network of nodes, which is used by an unknown-location node to determine its physical location. The Global Navigation Satellite System, GNSS (HofmannWellenhof, 2008) is an example of a widely used outdoor localization system. However, outdoor localization systems perform poorly in indoor environments due to strong signal attenuation and reflection by building materials, and no line-of-sight propagation. Thus, Indoor Localization Systems (ILSs) are needed to provide similar localization inside buildings. ILSs have many potential applications in the commercial, military and public safety sectors. This chapter focuses on the public safety application. The considered ILS is used to track emergency responders, e.g. fire-fighters and policemen, who carry out search and rescue missions in the disaster zone such as building fires and collapsed tunnels. Such an ILS was first crystallized in the EUROPCOM (Emergency Ultra wideband RadiO for Positioning and COMmunications) project (Harmer, 2008; Harmer et al., 2008). The EUROPCOM system is an ad hoc sensor network which comprises a small number of base or reference nodes deployed outside surrounding a building, and the rest of the nodes are unknown-location nodes which are worn and deployed by emergency responders entering the hostile building. The unknown-location node is self-localized by collectively determining its position relative to base nodes. Additionally, the unknown-location node is also allowed to determine its position relative to neighboring unknown-location nodes. This greatly enhances the accuracy and robustness of the ILS. It is fully autonomous and can be rapidly deployed with little human intervention.

Ultra-WideBand (UWB) is the radio transmission technology used by the EUROPCOM system. A UWB signal is defined to be one that possesses an absolute bandwidth of at least $500 \mathrm{MHz}$ or a fractional bandwidth larger than $20 \%$ of the center frequency. Currently, several UWB technologies exist, namely direct sequence UWB, impulse radio UWB, Multi- 
band Orthogonal Frequency Division Multiplexing (MB-OFDM) UWB, Chaotic UWB, and Frequency Hopping (FH-UWB). The EUROPCOM system selected FH-UWB because it offers significantly better range and position accuracy than other technologies such as pulse UWB (Frazer, 2004).

A great deal of effort has been expended on localization algorithms, but the Medium Access Control (MAC) and routing protocols for ILS have received very little attention yet. Unlike other ad hoc sensor networks, the considered ILS exhibits unique characteristics. Therefore, it poses new technical challenges in the MAC and multi-hop routing protocol design. Firstly, the ILS is heterogeneous in the sense it is composed of different types of nodes with varying capability, processing power and battery energy. Secondly, the ILS operates in a highly dynamic and hostile environment. Lastly, emergency applications require fast localization in the order of seconds. In order to address these challenges, we propose a novel SelfOrganizing Composite MAC (SOC-MAC) protocol and a Lightweight and robust Anycastbased Routing (LAR) protocol. Cross-layer approach is present in the design to attain highly optimized, bandwidth- and energy-efficient protocols.

\section{Network architecture of an Indoor Localization System (ILS)}

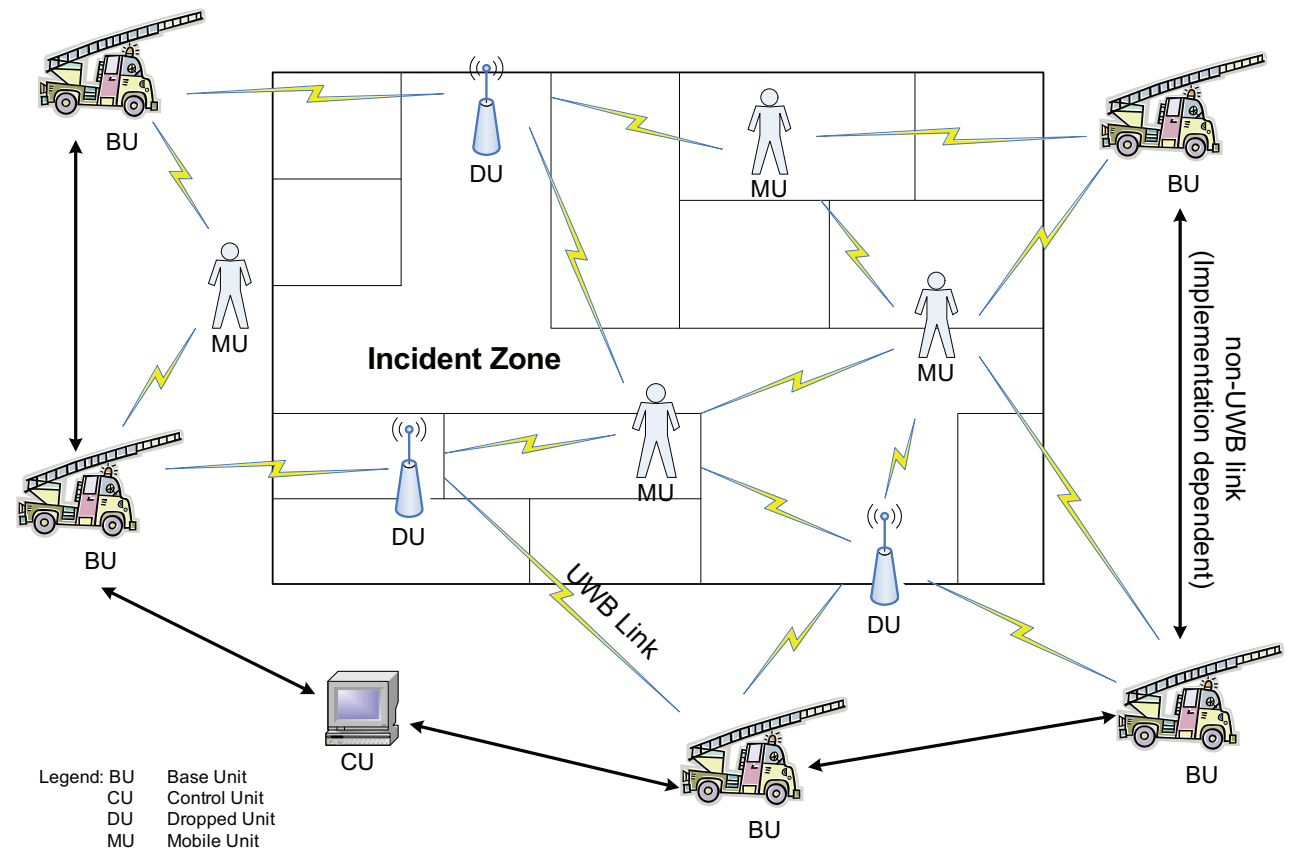

Fig. 1. Network Architecture of an Indoor Localization System

The assumed ILS, which is an ad hoc sensor network, consists of four types of nodes: a Control Unit (CU), Base Units (BUs), Dropped Units (DUs) and Mobile Units (MUs), as shown in Fig. 1. The MU is a sensor that is worn by every emergency responder. The MU has the capability to calculate its position which is in turn delivered to the CU. The BUs are located outside and around the incident area, while maintaining wireless connectivity with 
the emergency responders inside the building. Unlike other units, the position of BUs is known and most likely to be acquired through GNSS. Furthermore, the BUs will remain stationary throughout the entire mission. The DUs are strategically placed in the incident area by emergency responders to serve as relay nodes once the MUs lose wireless connectivity with the BUs. Similar to MUs, the DUs can determine their positions and relay them to the CU. The CU provides the main visual display to the rescue coordinators, showing the current position and direction of movement of individual emergency responders with respect to the incident area topology, e.g. a building. As shown in Fig. 1, the ILS is composed of a UWB subnetwork and a non-UWB subnetwork. The reason for two separate subnetworks is that the $\mathrm{CU}$ is not involved in the localization process. Thus, more radio resources are available for the UWB subnetwork, in particular, when the number of MUs increases.

\subsection{System assumptions}

In this subsection, we state several assumptions made in the design of the MAC and routing protocols. The MAC and routing protocol design assumes the FH-UWB technology is employed by the Physical layer of the BU, the DU and the MU. The operating bandwidth of the FH-UWB units is $1.25 \mathrm{GHz}$ which consists of 125 carrier frequencies. This means, the carrier spacing is $10 \mathrm{MHz}$. The center frequency is located at $5.1 \mathrm{GHz}$. Each unit follows a fixed hop pattern. The pair CU-BU communicates over a non-UWB link. Similarly, the BU$\mathrm{BU}$ transmission is also over non-UWB links. The rationale for using a non-FH-UWB technology is that more radio resources are available to the FH-UWB subnetwork. Since the non-FH-UWB technology is implementation-dependent, we will not further deal with the specifics of the non-UWB technology in the rest of the chapter. The design of the MAC and routing protocols is described in subsections 2.2 and 2.3, respectively.

\subsection{A Self-organizing Composite Medium Access Control (SOC-MAC) protocol}

As each MU is mobile, it will determine and transmit its position information to the $\mathrm{CU}$ periodically. For instance, in order to cope with user mobility in the order of $0.5 \mathrm{~m} / \mathrm{s}$ (walking speed), an MU needs to measure and transmit position information to CU at a rate of one position packet per second. As a result, SOC-MAC is based on the Time Division Multiple Access (TDMA) because such a MAC is particularly suited to the periodic nature of localization process. Unlike traditional TDMA, SOC-MAC is designed for ad hoc networks with no requirement for a central controller for allocating time slots as it is self-organizing.

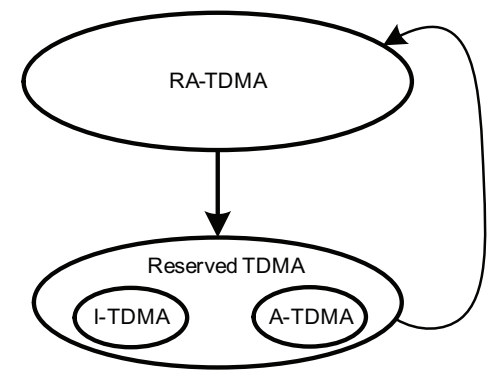

Fig. 2. SOC-MAC 
Using SOC-MAC, each unit can autonomously select and reserve time slots based only on local network knowledge without the need of dedicated signaling messages.

As shown in Fig. 2, the SOC-MAC protocol operates in two phases: a Random Access TDMA (RA-TDMA) phase and a Reserved TDMA phase. The former phase is invoked by a unit prior to joining the network or when the unit has not used any time slots in the previous superframe; thus, there are no reservations in the current frame. In this phase, a unit acquires a time slot through a random access mechanism. Once a time slot has been acquired, SOC-MAC will enter the Reserved TDMA phase. The RA-TDMA phase and the Reserved TDMA phase are described in the following subsections.

\subsubsection{Random Access TDMA (RA-TDMA) phase}

The UWB medium is segmented into SOC-MAC superframes, each of which has a constant period of $T$ seconds. Each superframe is in turn partitioned into $N$ orthogonal time slots of duration $T / N$ seconds. The start of the superframe is provided by one of the BUs, known as the Master BU (MBU). Naturally, MBU will occupy the first time slot. Units unable to hear the transmissions of MBU will synchronize to the TDMA frame by monitoring the transmissions of other neighboring units, which will identify the time slot in which they are transmitting. From this time slot number information, the start of the frame can be inferred.

Fig. 3 depicts the structure of the superframe and time slot. Each time slot can be used for either data transmission (referred to as "data slot") or ranging (referred to as "ranging slot"). The latter time slot format is specifically used for determining the range between two units. Thus, the ranging slot can accommodate a very limited payload. The MAC header includes identifiers of up to five ranging units, denoted as "R1 ID" to "R5 ID", which have been selected to respond to ranging requests. The final part of the ranging slot is reserved for the corresponding "pong" responses from "R1 ID" up to "R5 ID". Unlike ranging-slot, data-slot is purely utilized to transmit user data and can accommodate larger payload. The MAC header of the two slot structures contains similar fields except the ranging-slot includes the ranging unit identifiers and the position data of the transmitting unit (i.e., TX ID POS). Hence, the MAC header of the ranging-slot is longer and has to be split into two parts separated by two pilot tones as shown in Fig. 3.

In general a unit enters the RA-TDMA phase prior to joining the network. Fig. 4 contains the flow chart of the RA-TDMA phase. Before the unit can transmit in a time slot, it must listen to the physical channel for at least one complete TDMA superframe period. During this period, the unit constructs a list of one-hop neighbors and a map of their time-slot usage. Based on the time-slot usage map, the unit derives a list of vacant time slots in the forthcoming superframe. The number of vacant slots in the list is denoted as candidate slot counter (csc) in Fig. 4. When a first vacant time slot in the next superframe arrives, the $p$ persistent algorithm is applied to determine if this vacant time slot can be used for transmission. The $p$-persistent algorithm defines two parameters, namely $P 1$ and $P 2 . P 2$ is inversely proportional to $c s c$, and $P 1$ is randomly selected from an interval [0 .. 1]. If $P 1$ is equal to, or less than $P 2$, then the vacant time slot is reserved and transmission should occur in the reserved time slot. If not, the number of vacant time slots $\csc$ in the list is decremented by one and the same procedure is repeated for the next vacant time slot. The $p$-persistent algorithm minimizes the chance that two or more units in the RA-TDMA phase are contending for the same time slot. A low csc increases the probability of selecting the next vacant time slot. The number of unsuccessful attempts in reserving a time slot is recorded in attempt count $(a c)$. Once a vacant time slot has been successfully reserved, the RA-TDMA phase ends and the reserved TDMA phase sets in to complete the channel access procedure. 


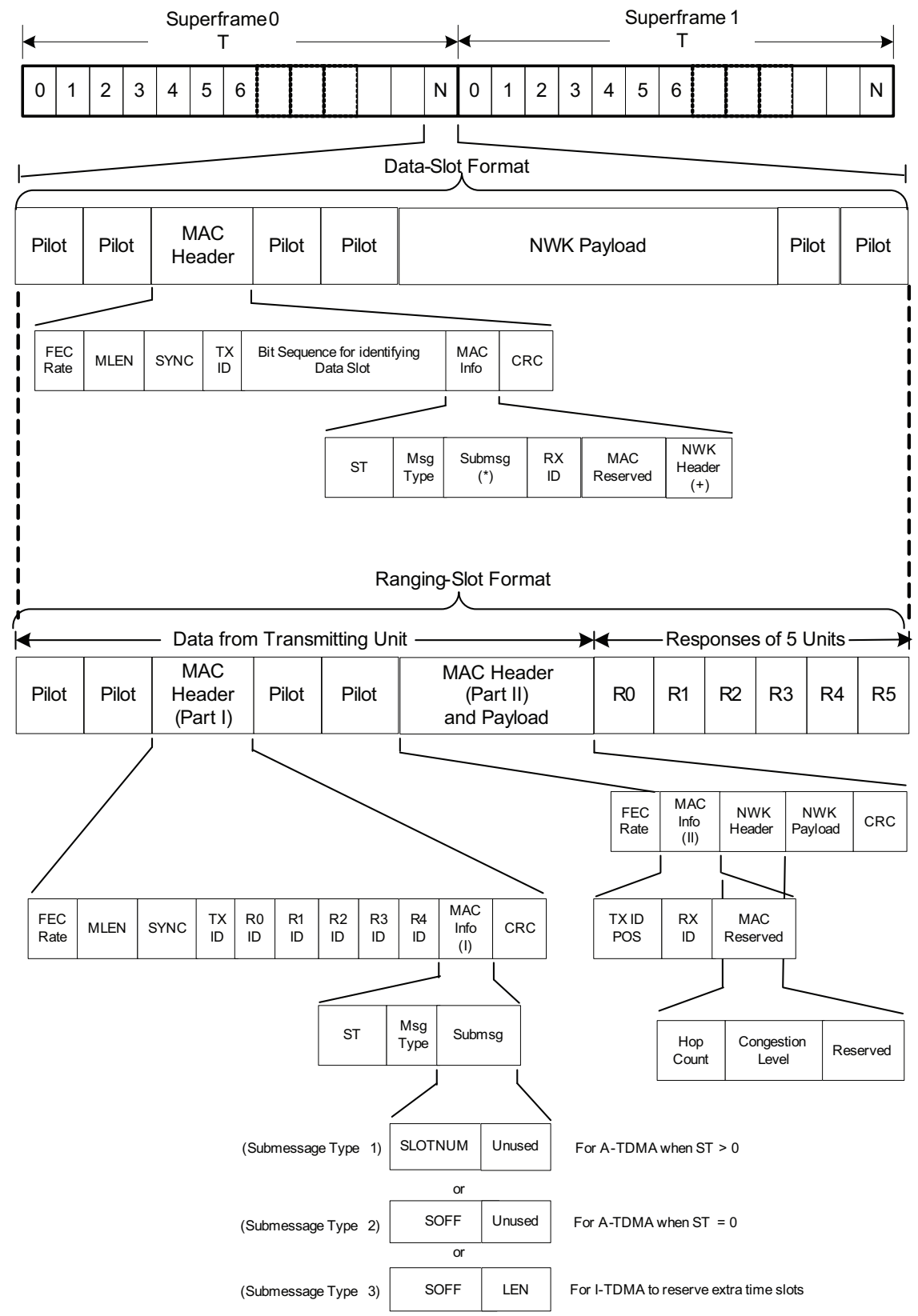

(*) This field has the same structure as the "submsg" field in the Ranging Slot

$(+)$ This field has the same structure as the NWK Header in the Ranging Slot

Fig. 3. SOC-MAC Superframe 


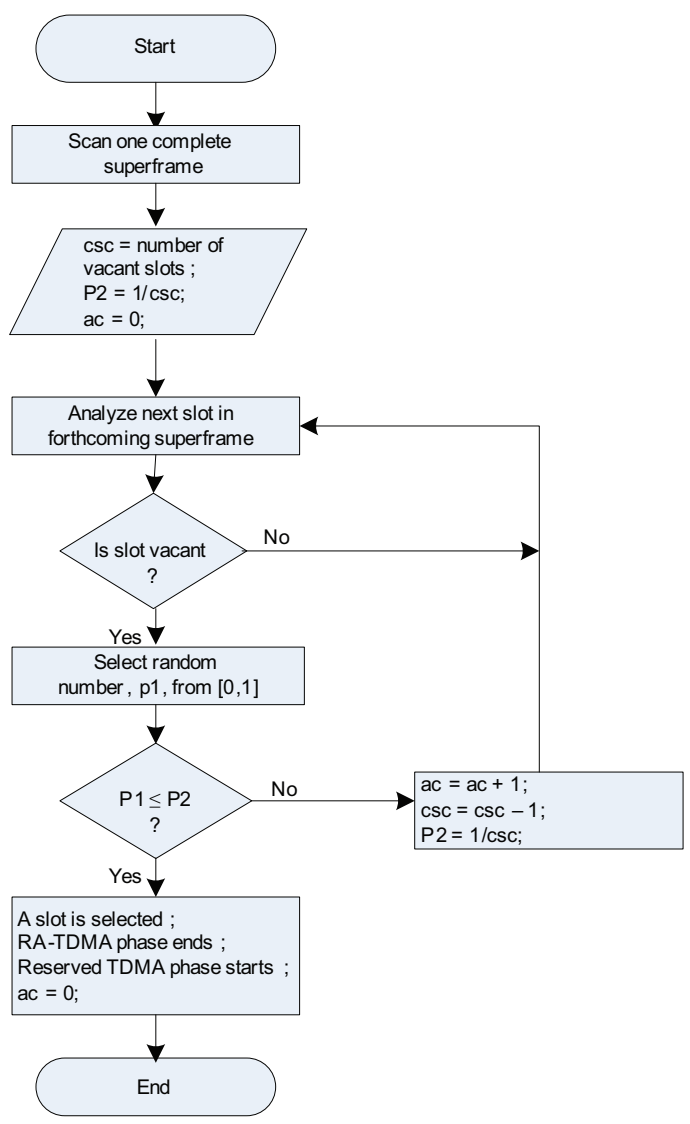

Fig. 4. Flow Chart of RA-TDMA

\subsubsection{Reserved TDMA phase}

The Reserved TDMA mode comprises two operations, namely the Autonomous TDMA (ATDMA) and the Incremental TDMA (I-TDMA). The latter is used to acquire additional slots in the same superframe in addition to the one acquired in the RA-TDMA phase. A-TDMA is responsible for managing the acquired time slots.

\section{A-TDMA}

Once a slot has been acquired through RA-TDMA and/or I-TDMA, the same time slot is automatically reserved for the next Slot_Timeout (ST) superframes, where ST is randomly picked from an interval [1 .. MAX_TIMEOUT]; MAX_TIMEOUT is a MAC design parameter. A-TDMA is responsible for keeping ST up-to-date. That is, ST is decremented by one in each new superframe. ST is included in the MAC header so that other units can determine when the time slot will be free. When ST $>0$, the Submessage Type 1 is used, which contains the time-slot number (SLOTNUM) of the currently reserved time slot as illustrated in Fig. 3. When a time slot expires (i.e., $\mathrm{ST}=0$ ), A-TDMA randomly chooses a vacant time slot in the next superframe from a list of vacant time slots in the time-slot usage map, and pre-announces to the other 
units the offset between the present time slot and the newly selected time slot (SOFF expressed in number of time slots) using the format Submessage Type 2 in the current superframe as shown in Fig. 3. This allows other units to find this unit in the next superframe without searching and to update the time-slot usage map. The new time slot will only be used in the next superframe. The continuous change of time-slot positions ensures that if two or more units had chosen the same time slot in the RA-TDMA phase, the collision can only persist for a maximum of MAX_Timeout superframes before one or all involved units must choose a different time slot. Thus, the collision is resolved through a probabilistic means. Hence, MAX_TIMEOUT must be small in order to reduce the number of collisions that are energy-wasting. On the other hand, if MAX_TIMEOUT is too small then neighbor units need to perform frequent updates on the time-slot usage map, which in turn increases power consumption. The new time slot is assigned a new ST value which is obtained using the same process as described above. The A-TDMA algorithm is depicted in Fig. 5.

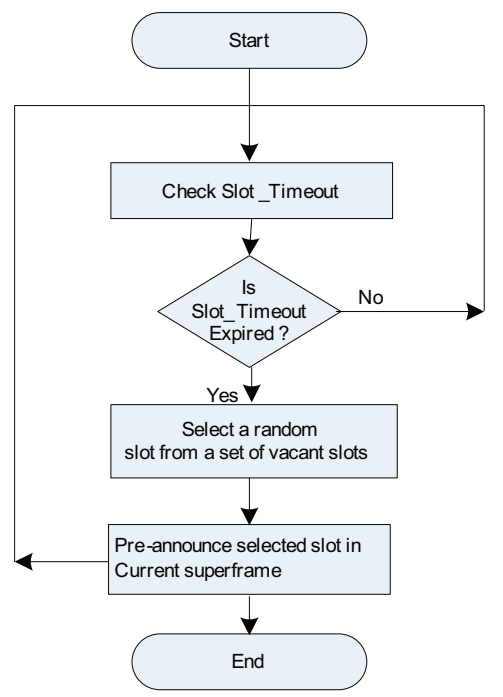

Fig. 5. Reserved TDMA Phase: ATDMA Operation

\section{I-TDMA}

The time slot acquired during the RA-TDMA phase is the first and only one for each unit. If a unit needs extra time slots, then I-TDMA is employed to reserve the extra time slots in the same superframe to increase the data rates. I-TDMA calculates the number of required time slots $N_{I}$ based on the actual queue length provided by the Network layer. It searches for a block of $N_{I}$ successive vacant time slots in the time-slot usage map. If not available, $N_{I}$ is reduced until the search is successful. The number of reserved time slots (LEN) and the offset (SOFF) between the current and the first new time slot are advertised using the format Submessage Type 3, refer to Fig. 3, so that all other units are informed about the new reservations. In principle each new time slot can be used for another I-TDMA operation so that the number of time-slot reservations can grow more rapidly. Hence, the usage of ITDMA needs to be restricted if the channel is busy and the number of vacant time slots is small. Note that in almost all cases the A-TDMA operation is required, while the I-TDMA 
operation is only sporadically needed to increase the data rates by reserving additional time slots. In order to free reserved time slots, the time slots are simply not renewed by A-TDMA after Slot_Timeout superframes.

\subsection{A Lightweight and Robust Anycast-based Routing (LAR) protocol}

The Lightweight and Robust Anycast-based Routing (LAR) protocol routes data packets from MUs or DUs to the nearest BU. There is no exact destination BU for a data packet. Thus, routing decisions must rely on routing parameters and packet types. LAR defines two routing parameters, namely hop count and congestion level. Hop count indicates the distance of a unit (in terms of the number of hops) to a reference BU. It increases monotonically at each hop. Congestion level is used to indicate the buffer occupancy of a unit. These routing parameters are not disseminated using dedicated routing packets but carried and propagated in the Network (NWK) header of data packets. Thus, LAR does not incur routing packet overheads. The format of the NWK header is depicted in Fig. 3. This means that irrespective of the data type, the NWK header always contains the mandatory routing parameters. The NWK header occupies 12 bits in a total of 1831 bits in one time slot of the SOC-MAC superframe. Therefore, the overheads of the NWK header for routing are less than $1 \%$, which conserves bandwidth and energy.

Route establishment is initiated by BUs to form spanning trees rooted at each BU. This is a natural choice because each BU periodically broadcasts its position which is known beforehand, while DUs and MUs just listen to the BU broadcasts since they need to determine their position. The BU sets the initial value for the hop count and congestion level. From the BU broadcasts, the DUs/MUs create a new entry in the routing table if it does not exist. The routing table entry contains the following fields: neighbor unit id, hop count, congestion level, FEC level and the expiration time of the entry. The first field identifies the address of the unit that broadcasts the data packet, which represent the next-hop unit for the route towards a destination BU. The neighbor unit id is contained in the MAC header. Note that the unit maintains only the next-hop routing state, which provides the routing protocol with a high degree of scalability. The hop count in the routing table is incremented by one with respect to the received hop count. For instance, if the incremented hop count is $n+1$ then the unit is $n+1$ hops away from the destination BU. The congestion level field is extracted from the NWK header. FEC (Forward Error Correction) level determines the channel bit rate for communicating with the next hop of the neighbor unit id. Four FEC levels, viz., FEC- 1 to FEC-4, are defined. FEC- 4 provides the highest bit rate but no or the lowest level of error protection. The FEC level is also contained in the MAC header. Once a DU/MU has determined its position, it can broadcast its position. The hop count in the NWK header is obtained from the selected route in the routing table entry, while the congestion is set to the maximum of its congestion level and that in the routing table entry. In the case of multiple entries in the routing table, a route selection algorithm with load balancing is used to choose the next hop. The algorithm will be described in subsection 2.3.1. So far, we have focused on route construction from an MU/DU to a destination BU, which is referred to as forward route. A reverse route (from a BU to an $\mathrm{MU}$ or DU) can be constructed using data packets sent on the forward route. One such data packet is position reporting which is used to transport position data to the BU. Position reporting packets are periodically sent by an MU and DU. The position reporting packets are transmitted using a forward route selected by the unit to a destination BU. All units along the forward route store the source and forwarding unit identifiers in their routing table. The latter identifier is 
the address of the intermediate unit that forwards the data packet while the source identifier is the address of the unit which generates the position reporting packets. No other routing parameters are needed for the reverse route. Since the position broadcasting and position reporting are periodic, the forward and reverse routes are always up-to-date. Therefore, no specific route recovery or maintenance functions are required.

\subsubsection{Load balancing}

Load balancing is achieved using the congestion level parameter, which is based on the occupancy of queues in a unit. The queues allocated by a unit are assumed to be fixed size. The congestion level is then deduced from the queue occupancy as shown in Table 1.

\begin{tabular}{|l|l|l|}
\hline Congestion Level & Queue Occupancy & \multicolumn{1}{c|}{ Definition } \\
\hline $0-2$ & $20 \%-40 \%$ full & Not congested \\
\hline $3-4$ & $50 \%-60 \%$ full & Slightly congested \\
\hline $5-6$ & $70 \%-80 \%$ full & Congested \\
\hline 7 & $90 \%$ full & Heavily congested \\
\hline
\end{tabular}

Table 1. Congestion Levels for Load Balancing

\subsubsection{Route selection}

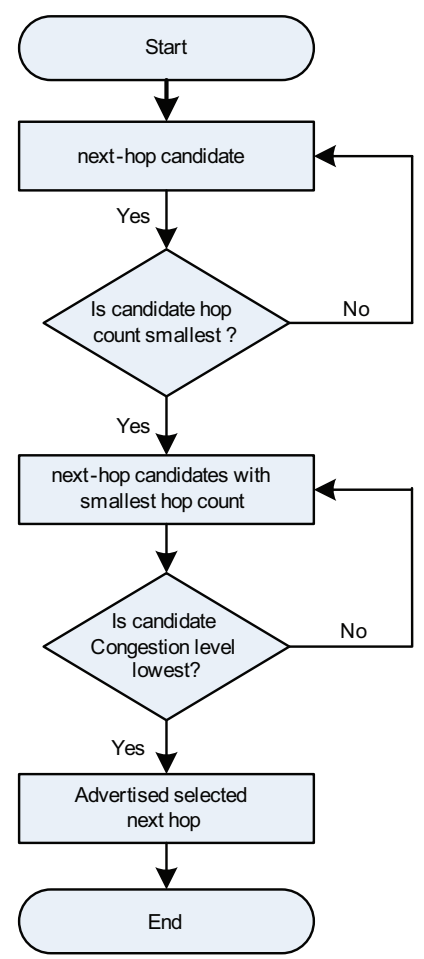

Fig. 6. Next-hop Selection Algorithm with Load Balancing 
Hop count is the primary routing metric, while congestion is the secondary metric due to the delay at the MAC layer, which cannot be tolerated by real-time data packets. In the case of multiple entries in the routing table, LAR must select the candidate route with the smallest hop count. If there are several candidate routes with the same hop count then the candidate with the lowest congestion level is picked. By selecting the candidate route with the smallest hop count the selection algorithm can guarantee loop-free delivery as a data packet is always forwarded from a unit with a higher hop count to a unit with lower hop count. The selection algorithm is shown in Fig. 6.

\section{Simulation set-up}

The feasibility and performance of SOC-MAC and LAR are evaluated by means of simulation. To this end, we extended the Mobility Framework (MF) (Mobility Framework) module by incorporating a model for a UWB Physical layer, the SOC-MAC protocol, the LAR protocol and the Application layer, and the ILS network entities. MF is an add-on package for simulating mobile and wireless networks on the OMNeT++ platform $(\mathrm{OMNeT}++)$ which is a powerful generic, object-oriented and discrete-event simulation tool. Naturally, MF can be easily extended for simulating the ILS network. Thus, three new simulation nodes, namely BUhost, DUhost, and MUhost, were defined. These nodes correspond to the units BU, DU and MU, respectively. CU was not modeled because it is in the non-UWB subnetwork which is implementation-specific. Fig. 7 depicts a sample of the simulation network, which consists of four BUhosts, two DUhosts and four MUhosts. In each of the simulation nodes, three protocol models, viz., the application, the network and the Network Interface Card (NIC) were defined as extensions to the corresponding models in MF. The internal structure of the node is shown in Fig. 8(a). The Blackboard and Mobility models were used without extensions. Note that BUhost, DUhost and MUhost have the same internal node structure. The application model, EuropAppLayer, the network model, EuropNetwLayer, the MAC model, EuropMacLayer, and the Physical model are described in the next subsections.

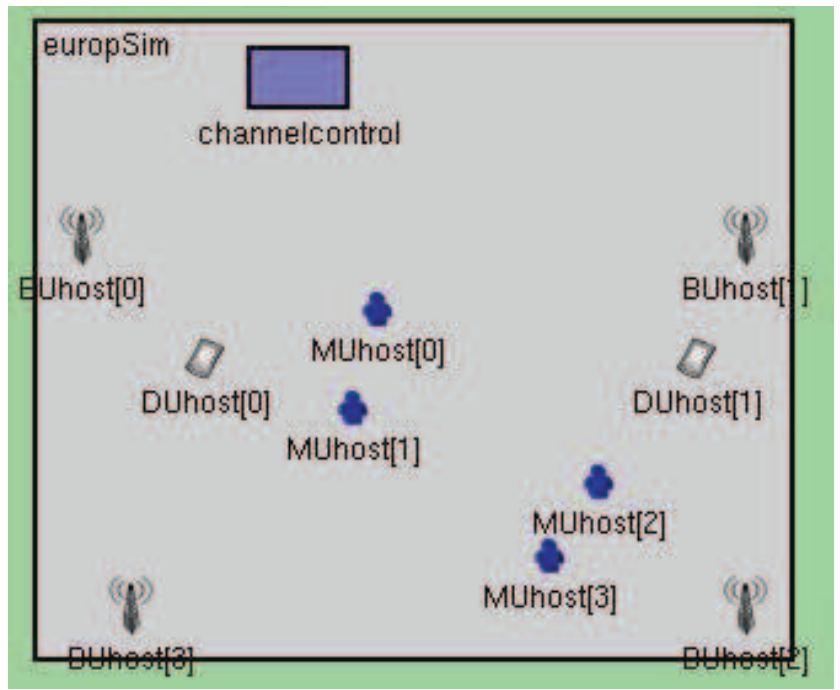

Fig. 7. Simulation Network 


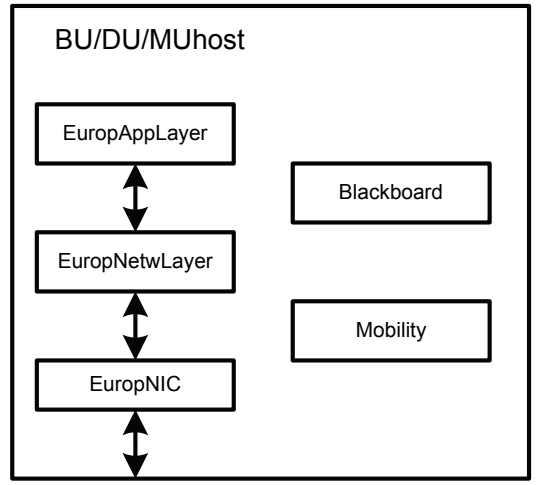

(a)

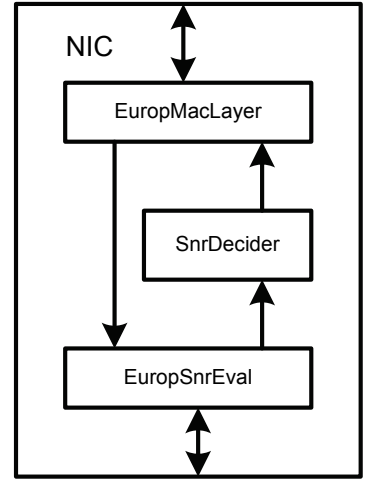

(b)

Fig. 8. Node and NIC Structure

\subsection{Physical layer model}

The Physical model is divided into EuropSnrEval and SnrDecider as shown in Fig. 8(b). The former was extended from SnrEval in MF while the latter was used as it is. EuropSnrEval is used to calculate the Signal-to-Interference-plus-Noise (SINR) of a received MAC frame. The SINR is defined as

$$
\operatorname{SINR}=10 \log \frac{P_{r}, \max }{P_{n}+I}
$$

where $P_{r, \max }$ is the strongest received signal power among the received signals, based on the capture effect (Rappaport, 2001). $P_{n}$ is the Additive White Gaussian Noise (AWGN). I is the interference power which is defined as the sum of all received signal power excluding $P_{r, \max }$. The interference power $I$ is expressed as

$$
I=\sum_{\neq P_{r, \max }} P_{r}
$$

In case of collision-free transmission, the term $I$ is null. Hence, Equation (1) is reduced to

$$
\operatorname{SINR}=10 \log \frac{P_{r}, \max }{P_{n}}
$$

The computed SINR is passed to SnrDecider which determines whether the MAC frame is correctly received or not. A MAC frame is considered to be correctly received, if SINR $\geq$ $\mathrm{SINR}_{\text {th }}$, where SINR $_{\text {th }}$ is the SINR threshold. A correctly received frame is delivered to the EuropMacLayer, otherwise it is discarded. SINR th $_{\text {was }}$ obtained through physical layer simulation, which produces Bit Error Rate (BER) plots as a function of SINR. Given a target

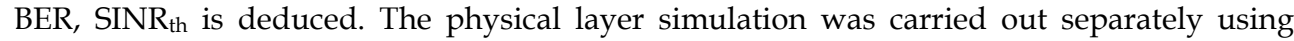
another tool since $\mathrm{OMNeT}++$ and $\mathrm{MF}$ lack the support for simulating physical layer functions such as frequency hopping, channel coding, modulation, and signal processing. 
The received power $P_{r}$ in Equation (1) is characterized by large-scale fading and small-scale fading. Large-scale fading represents the average signal power attenuation when transmitted through the medium. The attenuation or commonly known as Path Loss (PL) as a function of distance is expressed as (Rappaport, 2001).

$$
P L(d)=P L\left(d_{0}\right)\left(\frac{d}{d_{0}}\right)^{\gamma} 10^{x_{\sigma} / 10}, \quad d \geq d_{0}
$$

where $d_{0}$ is the reference distance, $\gamma$ is referred to the path loss exponent, and $X_{\sigma}$ denotes the log-normal shadowing effect with a zero-mean normal distribution (in $\mathrm{dB}$ ) and standard deviation $\sigma$ (also in $\mathrm{dB}$ ). $P L\left(d_{0}\right)$ is evaluated using the free-space path loss equation or by conducting measurements. In our work, $P L\left(d_{0}\right)$ was determined using the free-space path loss equation which is given by (Rappaport, 2001)

$$
P L\left(d_{0}\right)=\left(\frac{4 \pi f_{c} d_{0}}{c}\right)^{2}
$$

where $f_{c}=\frac{f_{\max }+f_{\min }}{2} . f_{\min }$ and $f_{\max }$ are the lower and upper boundary of UWB transmission frequency band, respectively. Substituting Equation (5) into Equation (4), and let $d_{0}=1 \mathrm{~m}$ in our case, we can rewrite Equation (4) as

$$
P L(d)=\left(\frac{4 \pi f_{c}}{c}\right)^{2} d^{\gamma} 10^{x_{\sigma} / 10}, \quad d \geq d_{0}
$$

Small-scale fading represents the wide variations in received signal strength caused by interference between two or more versions of the transmitted signal arriving at the receiver at slightly different times. It is typically modeled by the Ricean distribution or the Rayleigh distribution when there is a line-of-sight or non-line-of-sight, respectively. In UWB systems, the signal power variations due to small-scale fading are not severe due to the ultra-large bandwidth of UWB signals and diversity techniques used in the physical layer. Thus, in our physical channel model, we are only concerned with the large-scale fading. Hence, the received power $P_{r, \max }$ in Equation (1) and $P_{r}$ in Equation (2) can be calculated using

$$
P_{r}=\frac{P_{t}}{P L(d)}
$$

where $P L(d)$ is given in Equation (6), and $P_{t}$ is the transmit power.

\subsection{MAC layer model}

The MAC model, EuropMacLayer, captures the complete functionality of SOC-MAC described in Section 2.2. It was derived from the BasicMacLayer model of MF. The model definition consists of three parts, referred to as a EuropMacLayer module definition, a EuropMacLayer protocol data unit definition, and a EuropMacLayer module implementation. The EuropMacLayer module definition, which is specified using the OMNeT++ NED language. The EuropMacLayer protocol data unit definition, called EuropMacPkt, was derived from the MacPkt definition of MF. The derived module contains 
the fields of the EuropMacLayer protocol data unit only. The EuropMacLayer module implementation contains the algorithms of the composite MAC. Unlike the EuropMacLayer module definition and EuropMacPkt definition, this module was directly written in the C++ programming language. The EuropMacLayer module definition and EuropMacPkt are translated into $\mathrm{C}++$ code when an executable of the simulation program is built.

\subsection{Network layer model}

The Network model, EuropNetwLayer, implements the LAR protocol described in Section 2.3. It was derived from the SimpleNetwLayer model of MF. Similar to the MAC model, it consists of three parts: a EuropNetwLayer module definition, a EuropNetwLayer protocol data unit definition, and a EuropNetwLayer module implementation. The EuropNetwLayer protocol data unit definition, called EuropNetwPkt, was derived from the NetwPkt definition of MF.

\subsection{Application layer model}

The application traffic model generates dummy position packets of fixed size at regular intervals. The dummy position packets carry no real position information and the simulated nodes do not perform position estimation. This does not affect the performance of SOCMAC and LAR as long as the application model can mimic the traffic behavior of the real system. The application traffic model, called EuropApplLayer, which was derived from BasicApplLayer of MF. The application traffic model also consists of three parts: a EuropApplLayer module definition, a EuropApplLayer protocol data unit definition, and a EuropApplLayer module definition.

\section{Simulation results}

\subsection{SOC-MAC performance}

We analyze the performance of SOC-MAC. The performance measures for SOC-MAC are the successful SOC-MAC packet reception rate and the network throughput. An SOC-MAC packet consists of a header and payload for both the data- and ranging-slot as illustrated in Fig. 3. Thus, in one time slot, only one SOC-MAC packet is transmitted. The successful packet reception rate $P$ in the network is defined as the total number of SOC-MAC packets received by all units divided by the total number of SOC-MAC packets transmitted by all units in the network. Hence, $P$ is expressed as

$$
P=\frac{\sum_{i=1}^{M} r_{i}}{(M-1)\left(\sum_{j=1}^{M} b_{j}\right)}
$$

where $r_{i}$ is the number of MAC packets received by the $i$ th unit, and $b_{j}$ is the total number of MAC packets transmitted by the $j$ th unit. $M$ is the total number of units in the network. The scale factor in the denominator of Equation (8) is due to the fact that a packet transmitted by $j$ th unit is received by all the other $M-1$ units in the single hop case. Therefore, $P$ is unity in an ideal case.

Network throughput is defined as the total throughputs of all units, where the throughput of a unit is the amount of successfully received MAC frames in bits per second. The network 
throughput is normalized to the channel capacity. Thus, the normalized network throughput $S$ is defined as

$$
S=\frac{\sum_{i=1}^{M} x_{i}}{C}
$$

where $x_{i}$ is the throughput (in bits per second) of the $i$ th unit, $C$ is the channel capacity in bits per second, and $M$ is the total number of units in the network.

\begin{tabular}{|c|c|c|}
\hline \multicolumn{2}{|l|}{ Parameter } & Value \\
\hline \multicolumn{2}{|c|}{ Number of units $M=(\mathrm{MU}+\mathrm{DU}+\mathrm{BU})$} & From 10 to 360 \\
\hline \multicolumn{2}{|c|}{ Area $X \times Y \times Z$} & $40 \mathrm{~m} \times 40 \mathrm{~m} \times 3 \mathrm{~m}$ \\
\hline \multirow{5}{*}{ MAC } & Superframe duration $T$ & $4 \mathrm{~s}$ \\
\hline & Number of time slots $N$ & 160 \\
\hline & Time slot duration $\mathrm{T} / \mathrm{N}$ & $25 \mathrm{~ms}$ \\
\hline & $\begin{array}{l}\text { Number of time slots } \\
\text { allocated per Superframe }\end{array}$ & 1 and 4 time slots \\
\hline & MAX_TIMEOUT & $2,4,6,8,10$ \\
\hline \multirow{9}{*}{$\begin{array}{l}\text { Propagation } \\
\text { model }\end{array}$} & Transmit Power $P_{t}$ & $0.11 \mathrm{~mW}$ \\
\hline & AWGN $P_{n}$ & $-115.1 \mathrm{dBm}$ \\
\hline & Receiver Sensitivity & $-120 \mathrm{dBm}$ \\
\hline & Path loss exponent $\gamma$ & 3.5 \\
\hline & $\begin{array}{l}\text { Shadowing standard } \\
\text { deviation } \sigma\end{array}$ & $\begin{array}{l}0 \mathrm{~dB}, 2 \mathrm{~dB}, 4 \mathrm{~dB}, \\
8 \mathrm{~dB}\end{array}$ \\
\hline & $\mathrm{SINR}_{\text {th }}$ & $-5 \mathrm{~dB}$ \\
\hline & Bandwidth & $1.25 \mathrm{GHz}$ \\
\hline & $f_{\min }, f_{\max }$ & $6 \mathrm{GHz}, 7.25 \mathrm{GHz}$ \\
\hline & Center frequency $f_{c}$ & $6.625 \mathrm{GHz}$ \\
\hline \multicolumn{2}{|c|}{ Application Traffic model } & $\begin{array}{l}0.25 \text { packet/s, } \\
1 \mathrm{packet} / \mathrm{s}\end{array}$ \\
\hline \multicolumn{2}{|c|}{ Simulated time } & $600 \mathrm{~s}$ \\
\hline \multicolumn{2}{|c|}{ Number of simulation runs } & 30 \\
\hline
\end{tabular}

Table 1. Simulation Settings

The simulation parameter settings are given in Table 1 . In the simulation, the total number of units was varied from 10 to 360 units. Since we fixed the number of BUs to four units, the number of MUs and DUs was varied but always at an equal quantity. The DUs and MUs were randomly distributed in a square region with area $X \times Y \mathrm{~m}^{2}$. The length of $X$ and $Y$ is calculated such that signals can still be detected by the receiving units which are at the maximum distance from the transmitting units using the transmit power, AWGN, path loss

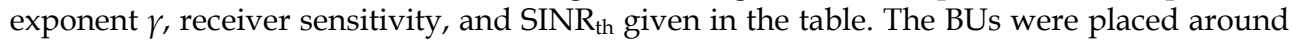
the edge of the area in order to reflect the arrangement of the real system. 


\subsubsection{Effects of shadowing}

Figs. 9 and 10 depict the successful MAC packet reception rate and network throughput as a function of the number of units without the shadowing effect, i.e., $\sigma=0 \mathrm{~dB}$. In an area of $40 \mathrm{~m} \times 40 \mathrm{~m}$, all units are within the radio transmission range of each other. This means that the transmission of a unit is heard by all the other $M-1$ units. In Figs. 9 and 10, two sets of similar simulation runs were carried out. In the first set, each unit reserved only one time slot in each SOC-MAC superframe, while in the second set, four time slots were reserved by each unit per SOC-MAC superframe. In the second set of the simulation run, the RA-TDMA phase is followed by I-TDMA to reserve the extra three time slots. The I-TDMA phase is not triggered in the first run since only one time slot is required, which is already reserved in the RA-TDMA phase. The plots for one time slot and four time slots, which are denoted as 1-timeslot (solid line) and 4-timeslot (dashed line), respectively in Figs. 9 and 10, exhibit similar behavior except the roll-off of the frame reception rate and the maximum network throughput occurs at different number of units. When the time slot occupancy is less than the number of time slots in a superframe, i.e., $N=160$, the network throughput increases linearly and the successful frame reception rate is $100 \%$. For $N=160$, the network can accommodate a maximum number of $M=160$ or 40 units for 1-timeslot and 4-timeslot, respectively. When $M=160$ or 40 , the successful reception rate drops to $90 \%$ for 1 -timeslot and 4-timeslot, respectively, and the network throughput reaches the peak, which is $90 \%$, as observed in Fig. 10 and then gradually falls as $M$ increases. When $M<40$ or 160, the effect of time slot collisions during the RA-TDMA phase is negligible. Thus collisions are resolved when the involved units enter the A-TDMA phase, which randomly picks a free time slot in the next superframe.

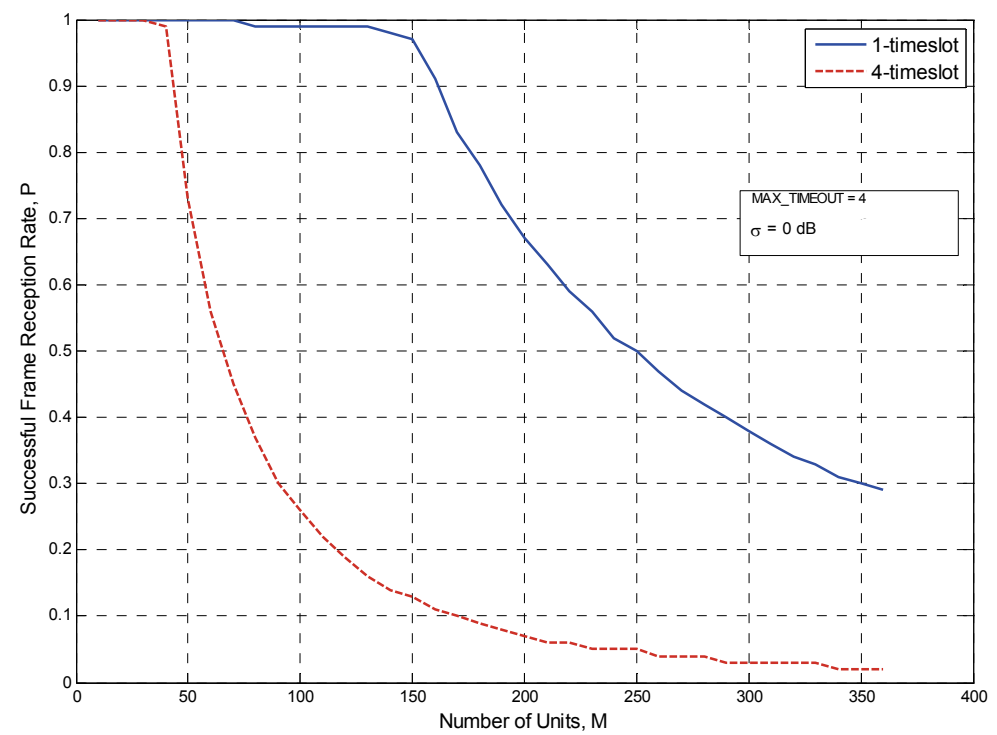

Fig. 9. Successful SOC-MAC Packet Reception Rate versus Number of Units for $\sigma=0 \mathrm{~dB}$, and MAX_TIMEOUT $=4$, and $\sigma=0 \mathrm{~dB}$ 


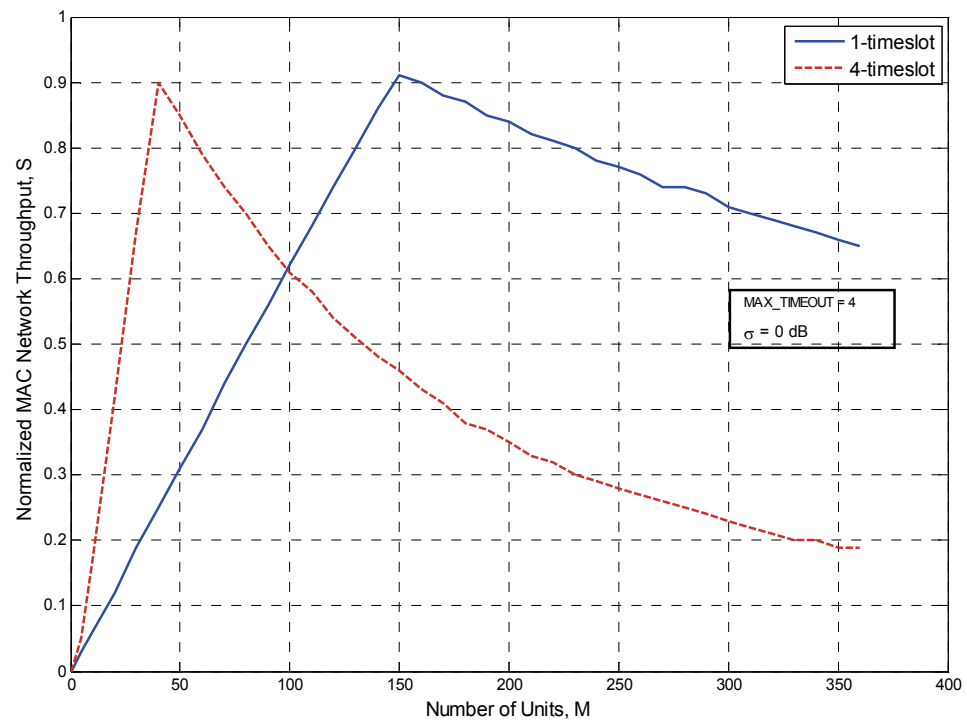

Fig. 10. Normalized Average SOC-MAC Network Throughput versus Number of Units for MAX_TIMEOUT $=4$, and $\sigma=0 \mathrm{~dB}$

When $M>40$ or 160 , both the successful frame reception rate and the network throughput rapidly deteriorate due to a larger number of collisions occurred in the RA-TDMA phase. Unlike in the case of $M \leq 40$ or 160, the A-TDMA is unable to resolve all the collisions because the number of time slots in the superframe is insufficient to accommodate the number of time slots needed by all the units. Simulation traces reveal that the collisions persisted through the entire simulation duration. In the case of collisions, the SINR of a received frame by all the receiving units is given by Equation (1). If the SINR of a received MAC frame is less than SINR $_{\text {th }}$ then the information on newly reserved time slot is lost in the collided time slot and it is considered to be free by other units. Hence, the set of free time slots built by each unit will consist of spurious free time slots since all the actual free slots are occupied. Furthermore, when the unit density is above the number of time slots in the superframe, the probability that two or more units select the false time slot is reasonably high.

Next, the same sets of simulation as above were repeated with the shadowing effect of $\sigma=2$ $\mathrm{dB}, 4 \mathrm{~dB}$ and $8 \mathrm{~dB}$. These values were chosen based on UWB channel measurements for indoor environment (Irahhauten et al., 2006). Figs. 11 and 12 show the successful SOC-MAC packet reception rate and the normalized network throughput as a function of the number of units. As shown in the figures, shadowing has detrimental effect on the performance. The performance degrades as $\sigma$ increases. For $\sigma=8 \mathrm{~dB}$, the maximum achievable network throughput is around $75 \%$ as compared to $90 \%$ for the case without shadowing. As observed in Fig. 12, the shadowing effect is more pronounced when $M=$ [35 .. 50] and [140 .. 200] for 4-timeslot and 1-timeslot, respectively. In this region of $M$, the channel capacity is saturated. Therefore, SOC-MAC packet losses are due to both collisions and shadowing. When $M>50$ and 200 for 4-timeslot and 1-timeslot, respectively, the majority of the SOCMAC packet losses are due to collisions rather than shadowing. This is evidenced by the convergence of the three plots. 


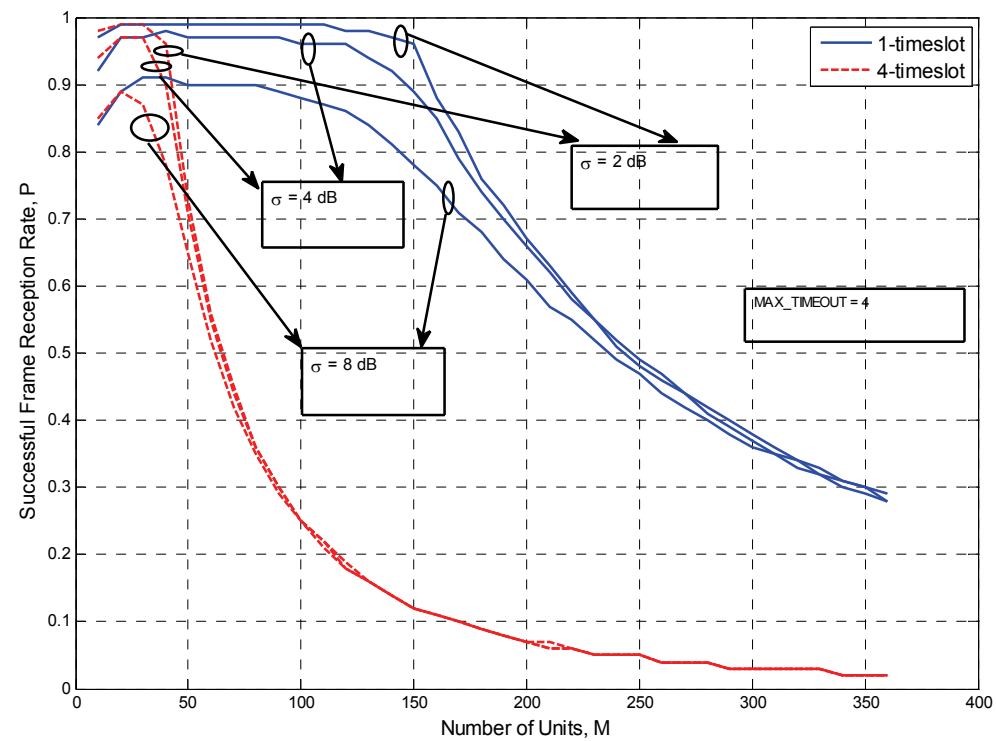

Fig. 11. Successful Packet Reception Rate versus Number of Units for MAX_TIMEOUT = 4, and $\sigma=2 \mathrm{~dB}, 4 \mathrm{~dB}$ and $8 \mathrm{~dB}$

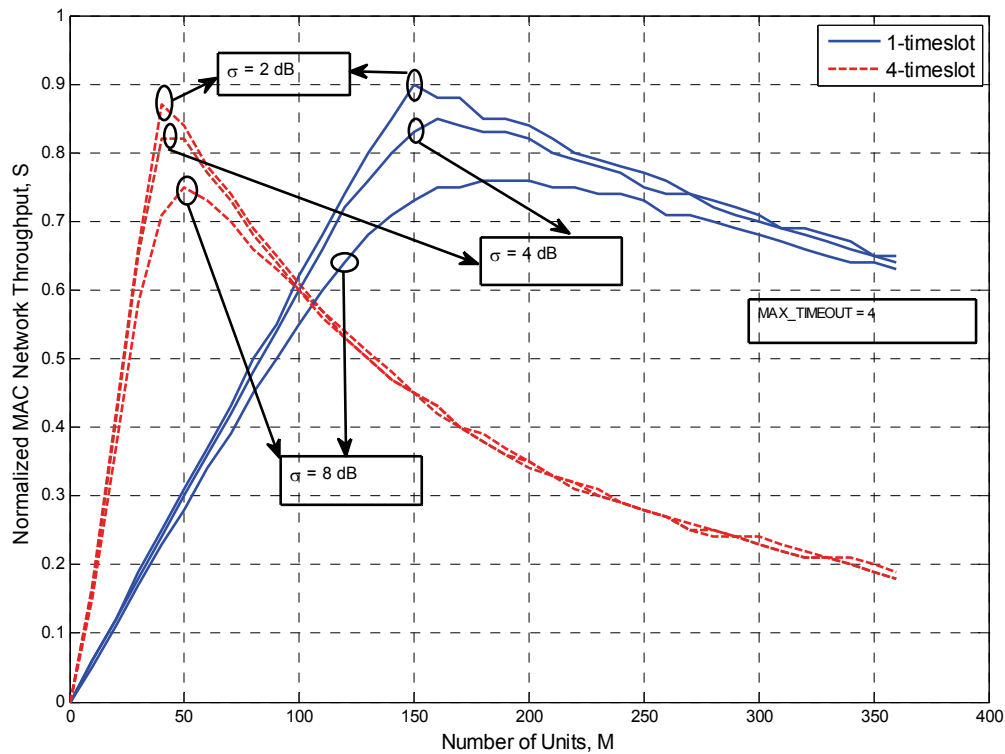

Fig. 12. Normalized Average SOC-MAC Throughput versus Number of Units for MAX_TIMEOUT $=4$, and $\sigma=2 \mathrm{~dB}, 4 \mathrm{~dB}$ and $8 \mathrm{~dB}$ 


\subsubsection{Effects of timeout}

In this subsection, we investigate the influence of the MAX_TIMEOUT parameter on the performance. As mentioned in subsection 2.2, MAX_TIMEOUT is the maximum number of superframes a unit may occupy a particular time slot. The same sets of simulation, as in subsection 4.1.1, were repeated and the MAX_TIMEOUT value was varied from 2 to 10 superframes in a step of 2. Figs. 13 and 14 depict the plots for the successful MAC packet reception rate and the network throughput. We can make two observations. Firstly, each of the MAX_TIMEOUT values delivers the same performance at low unit density, which is for $M<40$ and 150 for 4-timeslot and 1-timeslot, respectively. Secondly, at high unit density, larger MAX_TIMEOUT values give a slight performance advantage than smaller ones. The performance of MAX_TIMEOUT $=2$ is the worst, and MAX_TIMEOUT $=8$ and 10 achieve the best performance. For MAX_TIMEOUT $=2$, it means that the frequency of time slot renewal is the highest. Thus, at high unit density, a frequent renewal is not preferred because all of the time slots are fully occupied. If there is an available time slot, two or more units would select the same time slot, which results in collision as evidenced by the lowest successful MAC frame reception rate in Fig. 13. Additionally, information on the newly reserved time slot is lost in the collided time slot at high unit density. Other units would have mistaken these time slots for vacant. At high unit density, the time-slot occupancy map constructed by any unit would mainly consist of spurious time slots. As a result, selecting any of these spurious time slots would prolong collisions.

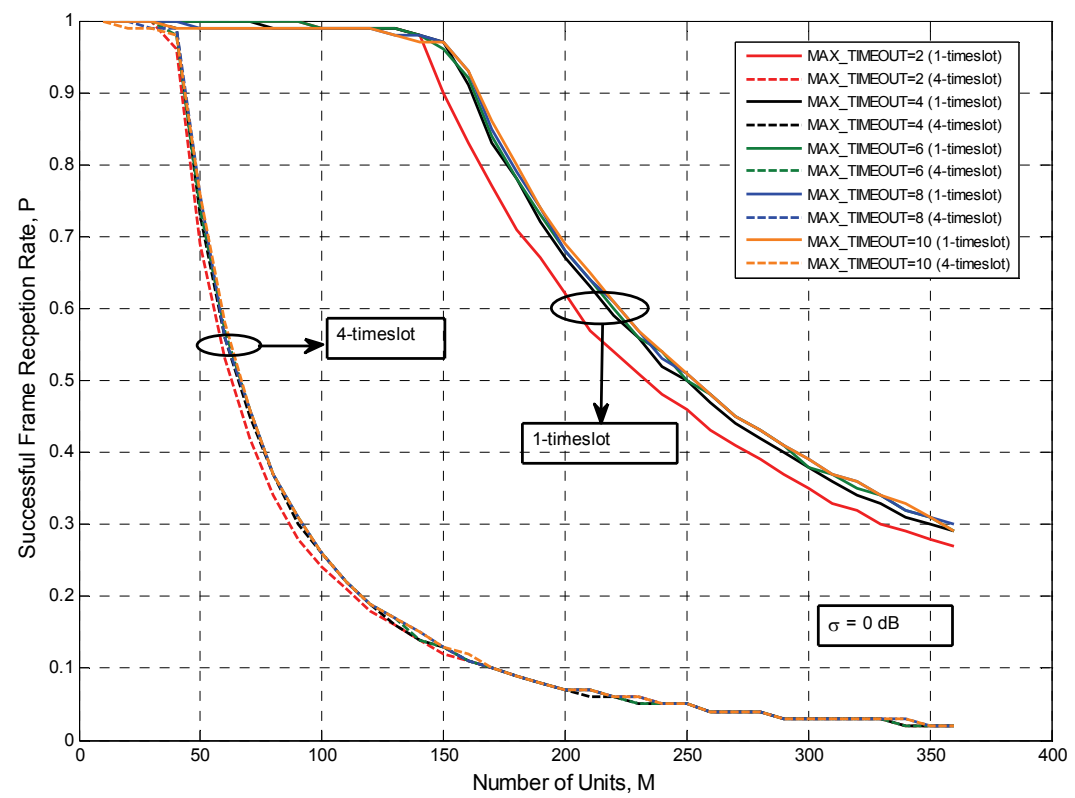

Fig. 13. Successful SOC-MAC Packet Reception Rate for $\sigma=0 \mathrm{~dB}$, and MAX_TIMEOUT $=[2$, $4,6,8,10]$ 


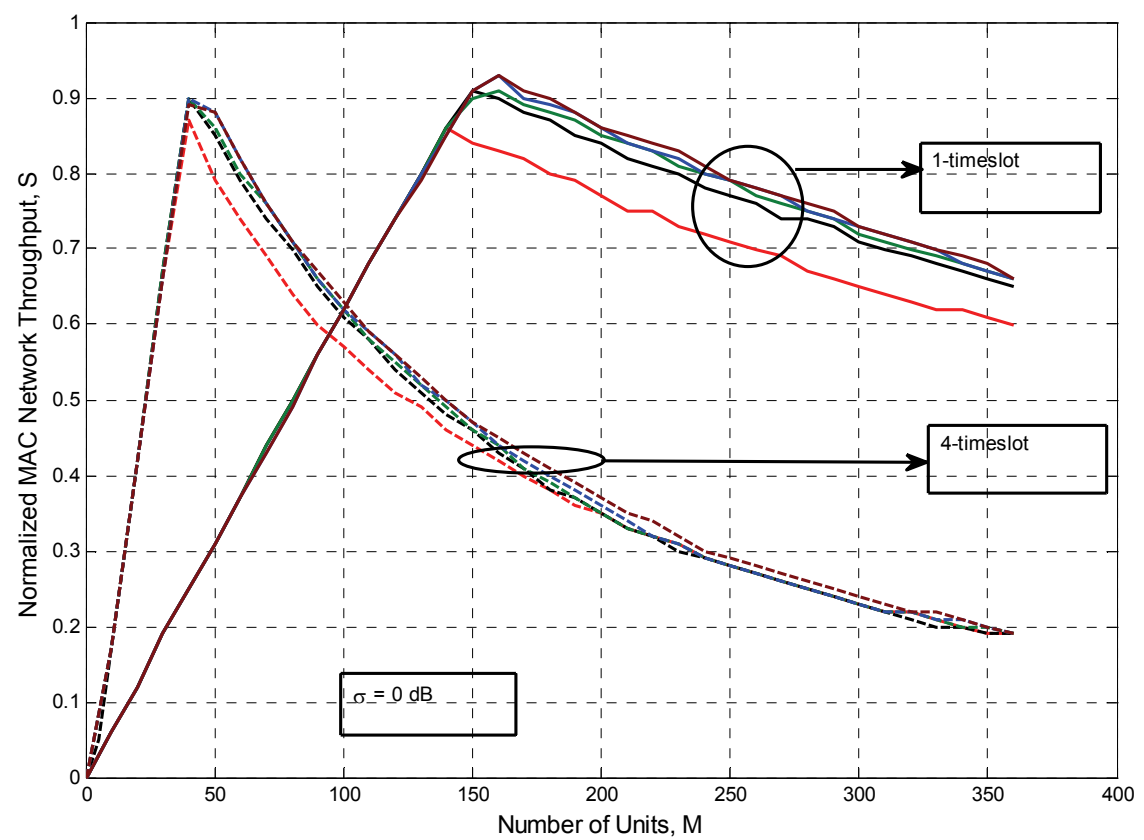

Fig. 14. Normalized Average SOC-MAC Network Througput for $\sigma=0 \mathrm{~dB}$, and MAX_TIMEOUT $=[2,4,6,8,10]$

\subsection{LAR performance}

In this subsection, we investigate the performance of LAR. First, we analyze the performance of LAR route establishment algorithm without the influence of route selection. Thus, a chain topology was used. With the chain topology, a unit usually has two neighbors except the unit at either end of the chain, which has only one. Each unit is stationary and spaced at an equidistant of $50 \mathrm{~m}$, which is just below the maximum transmission range that was determined using Equation (7) and the parameter values of Table 1. A single BU is placed at one end of the chain. A chain topology, which comprises $(h+1)$ units, consists of $h$ hops. In the chain topology, the BU is always assumed to be the 1st unit and the $(h+1)$ th unit is the last unit. The BU is responsible for initiating the route construction by broadcasting its position packets. The rest of the units in the chain are either MUs or DUs. The composition of MUs and DUs is irrelevant since they are functionally equivalent from the routing protocol point of view. The route discovery and end-to-end packet delays were examined. Route discovery delay is defined as the time in seconds when a unit (except the BU) found a route (i.e., next-hop neighbor) on the forward path. End-to-end delay is defined as the time in seconds taken by a data packet to traverse from an MU or a DU to the BU. Fig. 15 shows that both the route discovery and end-to-end packet delays are linearly proportional to the number of hops. The results prove that LAR became highly scalable. For a network diameter of 50 hops, it took the last unit at the end of the chain less than 30 SOC-MAC superframes to 
discover a route since the BU started broadcasting the routing information. End-to-end delays are determined using position reporting packets which are sent by the last unit, i.e. the $(h+1)$ th unit, to the BU for an $h$-hop network where $h$ varies from 1 to 50 . Note that the $(h+1)$ th unit only starts transmitting the position reporting packets once a route is found to remove the queuing effect due to route discovery.

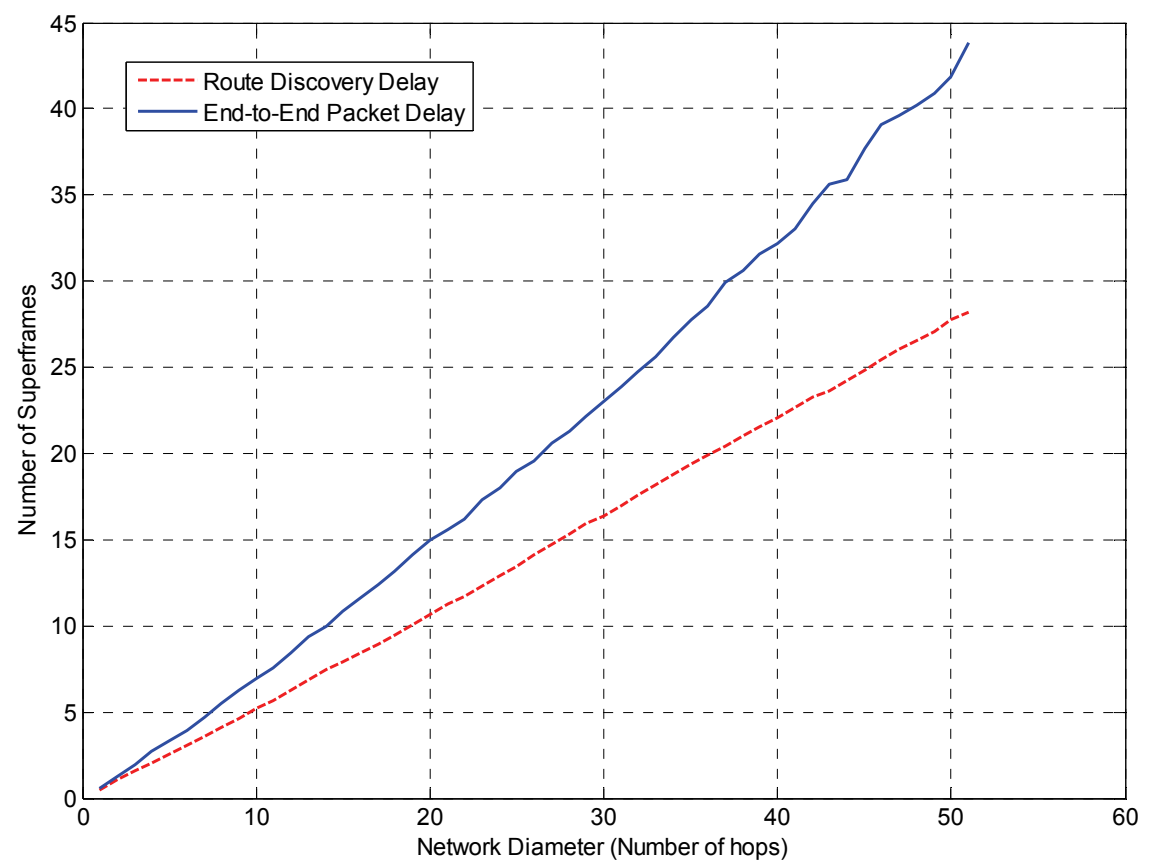

Fig. 15. Route discovery and End-to-End Packet Delay

Next, we will analyze the performance of the LAR route selection algorithm. For the analysis, we used a star topology as shown in Fig. 16. Each unit was stationary and spaced at an equidistant of 50m from its adjacent neighbors. BU0, BU1 and BU2 initiated the route construction simultaneously by broadcasting their position packets, and triggered neighboring units to transmit their positioning packets. In the star configuration, the unit at the center, MU0, received position packets from three different neighboring units, namely DU1, DU2 and MU1, see Fig. 16. Consequently, MU0 created three forward routes in its routing table. These routes are referred to as Route 1, Route 2 and Route 3, respectively, as shown in Fig. 16. The hop count of Route 1 and Route 2 is three hops while Route 3 is four. Since hop count is the primary routing metric, the routes with the least hop count would be selected by MU0. In this case, Route 1 and Route 2 were picked by the route selection algorithm of MU0. In the simulations, each unit broadcast position packets at a fixed interval of $4 \mathrm{~s}$. Hence, the traffic load was uniformly distributed across the network. In other words, none of the MUs or DUs were more congested than others. Therefore, the route 
selection algorithm would arbitrarily choose between Route 1 and Route 2 . The MU0 was set to transmit position reporting packet at time $t=50 \mathrm{~s}$ after the BUs started the route construction. Simulation traces show that Route 2 was selected by MU0 for transporting its position reporting packets to BU1. And the end-to-end packet delay is approximately 2 superframes, which conforms to the 3-hop delay in Fig. 15. At $t=100 s, M U 2$ was set to send position reporting packets, which introduced extra traffic on to the network. MU2 used Route 2 for transporting its position reporting packets since Route 2 was the shortest. Fig. 17 shows the congestion level seen by MU0.

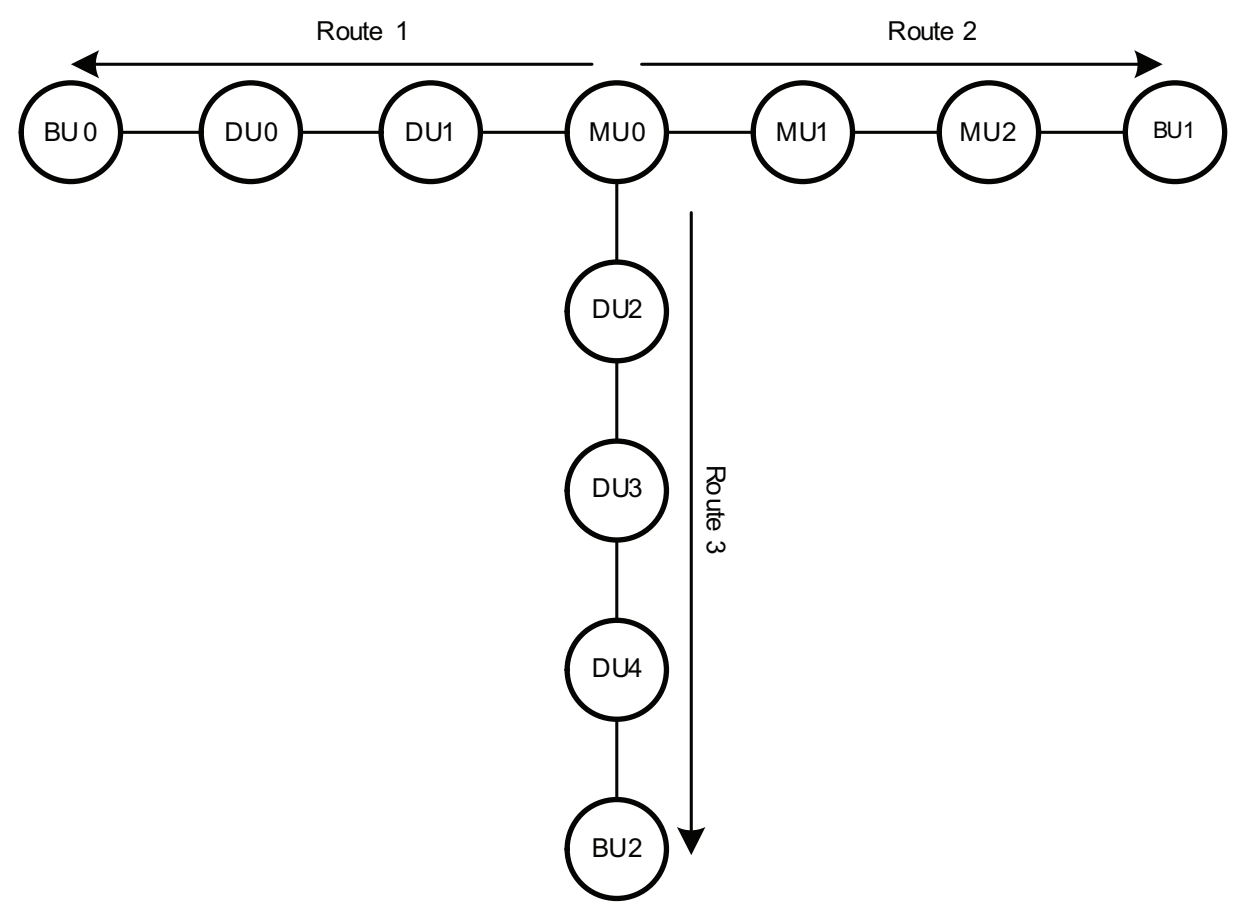

Fig. 16. Star Network Topology

Fig. 17 depicts the position reporting packets received by BU0 and BU1. As shown in Fig. 17, initially MU0 selected Route 2 for transporting its position reporting packets until the time was approximately 110s, where it switched to Route 1 . The switching occurred when MU0 detected the congestion level on Route 2 was increased to 3 . The increase in congestion was caused by MU2 when it started transmitting its position reporting packets at $t=100$ s. Due to congestion, some in-flight packets on Route 2 were experiencing excessive delays and arrived at BU1 later than packets sent on Route 1. The congestion level of both Route 1 and Route 2 continued to rise, and on Route 2, the congestion level reached the maximum at about 150s. When both MU0 and MU2 stopped transmitting position reporting packets at 250s, the congestion level did not drop until $t=350$ s for Route 2 and $t=410$ s for Route 1 because of a large number of packets already in the queue. At $t=350 \mathrm{~s}$, the congestion level 
of Route 2 dropped to 5, which was the same as Route 1 . At this point a route change occurred since MU0 selected Route 2 again. All the remaining packets in its queue were sent on Route 2 . After time $t=450$ s, the congestion level of both MU0 and MU2 dropped sharply.

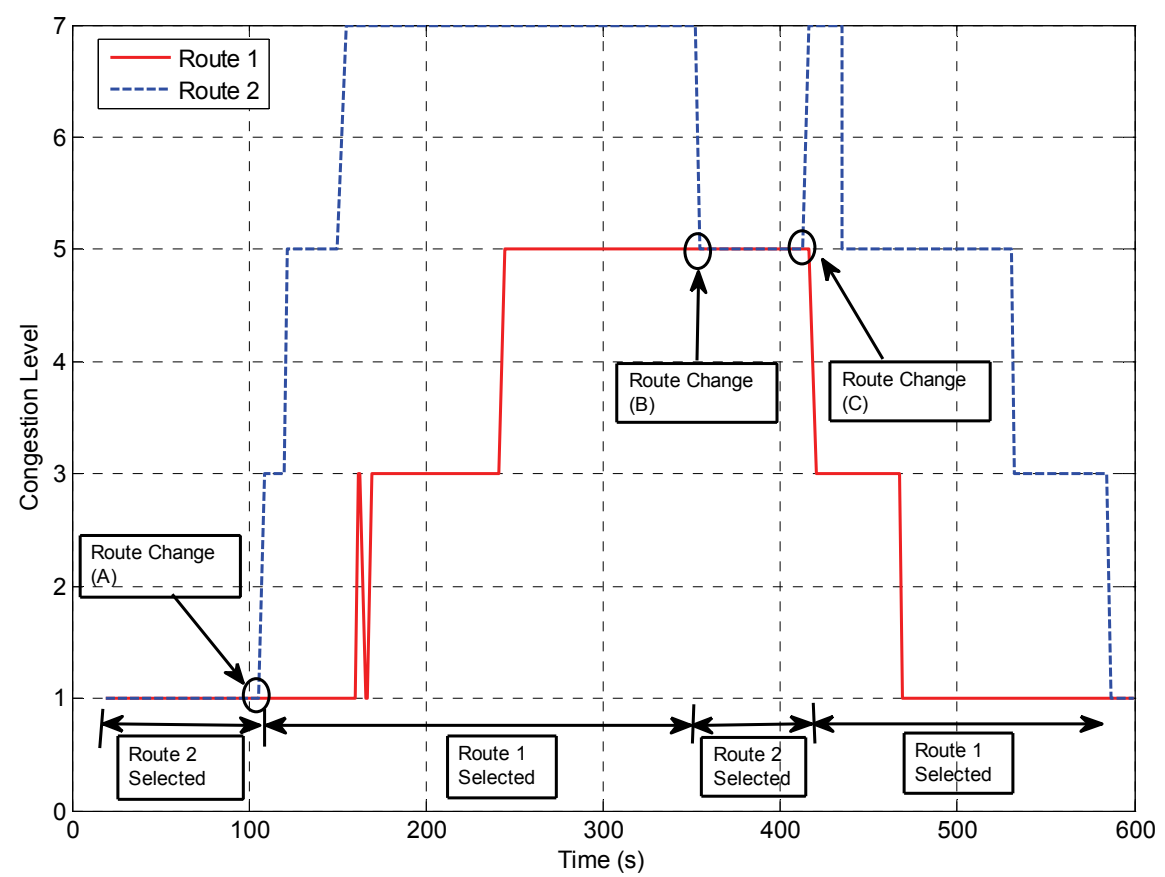

Fig. 17. Congestion Level

\section{Related work}

This section reviews the MAC and routing protocols developed for UWB-based ad hoc sensor networks.

\subsection{UWB-based MAC protocols for ad hoc sensor networks}

In the past few years, a number of MAC protocols have been proposed for UWB-based systems. (Legrand et al., 2003) and (Zhu \& Fapojuwo, 2005) proposed a modified version of the IEEE 802.15.3 Wireless Personal Area Network (WPAN) MAC protocol, which rely on a centralized controller. These MAC protocols can provide guaranteed Quality of Service (QoS) but are difficult to scale. The WHYLESS.COM project (Cuomo et al., 2002) proposed a distributed UWB MAC, which supports QoS and is scalable but has high complexity. (Chu \& Ganz, 2004) described a hybrid MAC for WPAN, which combines the advantages of both centralized and distributed protocols. The MAC protocol assumes that every node in a WPAN is one hop away from every other node. Consequently, the MAC is foreseen to face 
scalability issues when operating in multi-hop scenarios. Furthermore, a separate control channel is used for signaling purposes, which increases the complexity and is not lightweight for low bit-rate channels. Ultra-Wideband MAC (U-MAC) (Jurdak et al., 2005) is a proactive and adaptive protocol. Similar to (Chu \& Ganz, 2004), a separate signaling channel is needed for exchanging a node's state information with its direct neighbors. (Broutis et al., 2007) and (Benedetto et al., 2005) outlined a multi-channel MAC in which communication between two nodes takes place on orthogonal channels. The complexity and overheads incurred by such a MAC protocol are higher than single-channel MAC protocols. (Merz et al., 2005) proposed a combined Physical and MAC layer for very low power UWB system. No separate control channel is needed. However, the signaling overheads incurred by the MAC can be significant for short data packets and low bit-rate channels. In summary, all of the above-mentioned MAC protocols were not designed for localization application in mind. The IEEE 802.15.4a standard (Karapistoli et al., 2010; IEEE 802.15.4a, 2007) specifies a Physical layer and a MAC layer which support localization. The IEEE 802.15.4a MAC supports two different modes of channel access: beacon-enabled and nonbeacon-enabled. The latter is suited for localization application. Unlike SOC-MAC, the nonbeacon-enabled mode of the IEEE 802.15.4a MAC is based on the classical Aloha scheme or the CSMA/CA scheme.

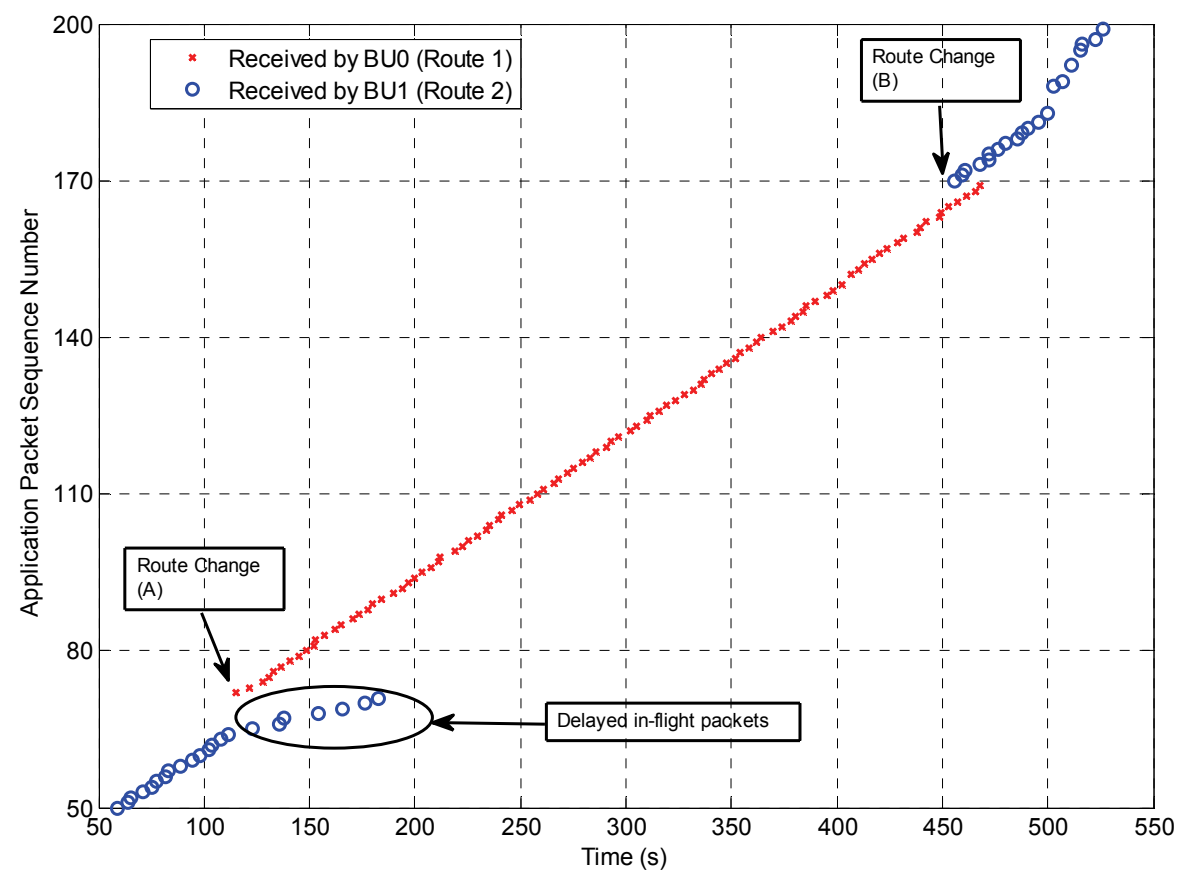

Fig. 18. Position Reporting Packets 


\subsection{Routing protocols for ad hoc sensor networks}

A large number of routing protocols, e.g. (Kulik et al., 2002; Intanagonwiwat et al., 2000; Schurgers \& Srivastava, 2001; Shah \& Rabaey, 2002; Lindsey \& Raghavendra, 2002; Manjeshwar \& Agarwal, 2001), have been developed for ad hoc sensor networks. Although the considered ILS is an ad hoc sensor network, it has some profound distinctions which mean existing ad hoc sensor routing protocols are not directly applicable. Firstly, sensor nodes are generally assumed to have very low mobility after deployment (Al-Karaki \& Kamal, 2004) in comparison with ILS. Lastly, the relative size of ad hoc sensor networks is huge in the order from thousands to millions of nodes (Al-Karaki \& Kamal, 2004) as compared to ILS.

\section{Summary}

In this chapter, we described the SOC-MAC and LAR protocols that are tailored for indoor localization systems used to track emergency responders. The cross-layer approach is present in the protocol design in order to optimize bandwidth and battery-energy consumption. As a result, SOC-MAC is simple and self-organizing, which is composed of two phases, namely RA-TDMA and reserved TDMA. The former is for initial acquisition of time slots while the latter is for management and maintenance of time slots. In addition to simplicity, LAR is extremely lightweight. No dedicated routing packets are needed. Instead, routing information is carried in the network header of localization packets, which constitutes less than $1 \%$ of the total channel capacity. We validated and studied the performance of SOC-MAC and LAR by simulations under varying SOC-MAC and LAR parameters.

\section{Acknowledgement}

The work was partially funded by the IST-004154 EUROPCOM project.

\section{References}

Al-Karaki, J. N. \& Kamal, A. E. (2004). Routing Techniques in Wireless Sensor Networks: A Survey, IEEE Wireless Communications Magazine, Vol. 11, No. 6

Benedetto, M.-G.; De Nardis, L.; Junk, M. \& Giancola, G. (2005). (UWB)2: Uncoordinated, Wireless, Baseborn Medium Access for UWB Communication Networks, Mobile Networks and Applications (MONET), Vol. 10, No. 5

Broutis, I.; Krishnamurthy, S. V.; Faloutsos, M.; Molle, M. \& Forester, J. R. (2007). Multiband Media Access Control in Impulse-based UWB Ad Hoc Networks, IEEE Transactions on Mobile Computing, Vol. 6, No. 4

Chu, Y. \& Ganz, A. (2004). MAC Protocols for Multimedia Supporting UWB-based Wireless Networks, Proceedings of 1st Int'l Conference on Broadband Networks (BROADNETS)

Cuomo, F.; Martello, C.; Baiocchi, A. \& Fabrizio, C. (2002). Radio Resource for Ad Hoc Networking with UWB, IEEE Journal on Selected Areas in Communications, Vol. 20, No. 9 
Frazer, E. L. \& Tee, D. (2004). A Comparison of UWB Technologies for Indoor Positioning as an Augmentation to GNSS, Proceedings of 2nd European Space Agency (ESA) Workshop on Satellite Navigation User Equipment Technologies (NAVITEC), Noordwijk, The Netherlands, 2004

Harmer, D. (2008). EUROPCOM: Ultra-WideBand Radio for Rescue Services, Proceedings of 2nd Int'l Workshop on Robotics for Risky Interventions and Surveillance of the Environment (RISE), Benicassim, Spain, 2008

Harmer, D., et al. (2008). EUROPCOM: Emergency Ultra-WideBand (UWB) Radio for Positioning and Communications, Proceedings of IEEE International Conference on Ultra-WideBand (ICUWB), 2008

Hofmann-Wellenhof, B.; Lichtenegger, H. \& Wasle, E. (2008). GNSS - Global Navigation Satellite Systems: GPS, GLONASS, and more, Springer, Vienna

IEEE 802.15.4a (2007). Wireless Medium Access Control (MAC) and Physical Layer (PHY) Specifications for Low-Rate Wireless Personal Area Networks (WPANs)

Intanagonwiwat, C.; Govindan, R. \& Estrin, D. (2000). Directed Diffusion: a Scalable and Robust Communication Paradigm for Sensor Networks, Proceedings of ACM MobiCom, Boston, MA, 2000

Irahhauten, Z.; Janssen, G. J. M., Nikookar, H., Yaravoy, A. \& Lighart, L. P. (2006). UWB Channel Measurements and Results for Office and Industrial Environments, Proceedings of Int'l Conference on Ultra-WideBand (ICUWB), MA, 2006

Jurdak, R.; Baldi, P. \& Lopes, C. V. (2005). U-MAC: A Proactive and Adaptive UWB Medium Access Control Protocol, Wiley Wireless Communications and Mobile Computing, Vol. 5 , No. 5

Karapistoli, E.; Pavlidou, F.; Gragopoulos, I. \& Tsetsinas, I. (2010). An Overview of the IEEE 802.15.4a Standard, IEEE Communications Magazine, Vol. 48, No. 1

Kulik, J.; Heinzelman, W. R. \& Balakrishnan, H. (2002). Negotiation-based Protocols for Disseminating Information in Wireless Sensor Networks, Wireless Networks, Vol. 8

Legrand, J.; Bucaille, I.; Hethuin, S.; De Nardis, L.; Giancola, G.; Di Benedetto, M.; Blazevic, L. \& Rouzet, P. (2003). U.C.A.N.'s Ultra Wideband Medium Access Control Schemes, Proceedings of Int'l Workshop on Ultra Wideband Systems (IWUWBS), 2001

Lindsey, S. \& Raghavendra, C. (2002). PEGASIS: Power-efficient Gathering in Sensor Information Systems, Proceedings of Aerospace Conference, 2002

Manjeshwar, A. \& Agarwal, D. P. (2001). TEEN: a Routing Protocol for Enhanced Efficiency in Wireless Sensor Networks, 1st Int'l Workshop on Parallel and Distributed Computer Issues in Wireless Networks and Mobile Computing, 2001

Merz, R.; Widmer, J.; Le Boudec, J. Y. \& Radunovic, B. (2005). A Joint PHY/MAC Architecture for Low Radiated Power TH-UWB Wireless Ad Hoc Networks, Wiley Wireless Communications and Mobile Computing, Vol. 5, No. 5

Mobility Framework, http://mobility-fw.sourceforge.net

OMNeT++, http://www.omnetpp.org/

Rappaport, T. (2001). Wireless Communications, 2nd edition, Prentice Hall

Schurgers, C. \& Srivastava, (2001). Energy-efficient Routing in Wireless Sensor Networks, MILCOM Proceedings on Communications for Network-Centric Operations: Creating the Information Force, McLean, VA, 2001 
Shah, R. C. \& Rabaey, J. (2002). Energy Aware Routing for Low Energy Ad Hoc Sensor Networks, Proceedings of WCNC, Orlando, FL, 2002

Zhu, J. \& Fapojuwo, A. O. (2005). A Complementary Code-CDMA-based MAC Protocol for UWB WPAN System, EURASIP Journal on Wireless Communications and Networking, Vol. 2005, No. 2 


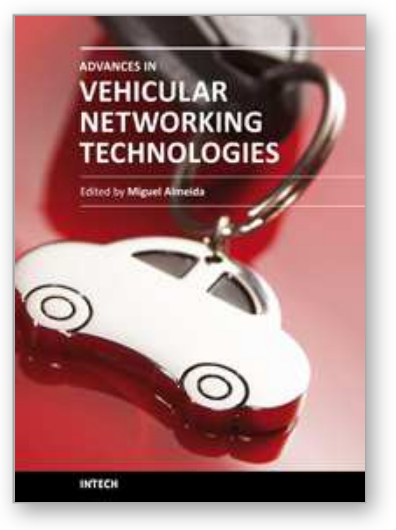

\author{
Advances in Vehicular Networking Technologies \\ Edited by Dr Miguel Almeida
}

ISBN 978-953-307-241-8

Hard cover, 432 pages

Publisher InTech

Published online 11, April, 2011

Published in print edition April, 2011

This book provides an insight on both the challenges and the technological solutions of several approaches, which allow connecting vehicles between each other and with the network. It underlines the trends on networking capabilities and their issues, further focusing on the MAC and Physical layer challenges. Ranging from the advances on radio access technologies to intelligent mechanisms deployed to enhance cooperative communications, cognitive radio and multiple antenna systems have been given particular highlight.

\title{
How to reference
}

In order to correctly reference this scholarly work, feel free to copy and paste the following:

Anthony Lo, Alexander Yarovoy, Timothy Bauge, Mark Russell, Dave Harmer and Birgit Kull (2011). An UltraWideband (UWB) Ad Hoc Sensor Network for Real-time Indoor Localization of Emergency Responders, Advances in Vehicular Networking Technologies, Dr Miguel Almeida (Ed.), ISBN: 978-953-307-241-8, InTech, Available from: http://www.intechopen.com/books/advances-in-vehicular-networking-technologies/an-ultrawideband-uwb-ad-hoc-sensor-network-for-real-time-indoor-localization-of-emergency-responder

\section{INTECH}

open science | open minds

\section{InTech Europe}

University Campus STeP Ri

Slavka Krautzeka 83/A

51000 Rijeka, Croatia

Phone: +385 (51) 770447

Fax: +385 (51) 686166

www.intechopen.com

\section{InTech China}

Unit 405, Office Block, Hotel Equatorial Shanghai

No.65, Yan An Road (West), Shanghai, 200040, China

中国上海市延安西路65号上海国际贵都大饭店办公楼 405 单元

Phone: +86-21-62489820

Fax: +86-21-62489821 
(C) 2011 The Author(s). Licensee IntechOpen. This chapter is distributed under the terms of the Creative Commons Attribution-NonCommercialShareAlike-3.0 License, which permits use, distribution and reproduction for non-commercial purposes, provided the original is properly cited and derivative works building on this content are distributed under the same license. 